Para enlazar con este artículo / To link to this article: http://dx.doi.org/10.6035/MonTI.2019.11.1

Para citar este artículo / To cite this article:

Tolosa Igualada, Miguel \& Álvaro Echeverri. (2019) "Porque algo tiene que cambiar. La formación de traductores e intérpretes: Pasado, presente y futuro.” En: Tolosa Igualada, Miguel \& Álvaro Echeverri (eds.) 2019. Porque algo tiene que cambiar. La formación de traductores e intérpretes: Presente \& futuro / Because something should change: Present \& Future Training of Translators and Interpreters. MonTI 11, pp. 9-28.

\title{
PORQUE ALGO TIENE QUE CAMBIAR. LA FORMACIÓN DE TRADUCTORES E INTÉRPRETES: PASADO, PRESENTE Y FUTURO
}

\author{
Miguel Tolosa Igualada \\ Miguel.tolosa@ua.es \\ Universidad de Alicante \\ Álvaro Echeverri \\ a.echeverri@umontreal.ca \\ Universidad de Montreal
}

\section{Resumen}

La formación de intérpretes y traductores ha ido experimentando cambios importantes. De las metodologías centradas en el profesor como el "apprenticeship approach", en interpretación o el método de "¿quién lee la siguiente frase?", en traducción se ha ido pasando lentamente a enfoques situados que priorizan procesos cognitivos superiores en situaciones auténticas. Los avances del discurso sobre la pedagogía de la traducción y los progresos logrados en materia de investigación en interpretación han permitido la consolidación de dos comunidades con una fuerte intensidad investigativa. El objetivo de este artículo es presentar una retrospectiva del desarrollo de la formación de intérpretes y de traductores y proponer algunas ideas para adaptar la formación a la forma de aprender de los "nativos digitales".

\begin{abstract}
"Because Something Should Change. Translator and Interpreter Training: Past, Present and Future"

The interpreter and translator training has undergone important changes. The methodologies centred on the teacher (i.e. "apprenticeship approach", in interpretation or the method "who takes the next sentence?" in translation) have been slowly moving towards situated approaches which foster higher cognitive processes in the framework of authentic situations. The advances and progress in the pedagogy of translation and
\end{abstract}


interpreting field have led to the consolidation of two communities with a strong investigative intensity. The aim of this article is to present a retrospective of the development of the training of interpreters and translators and to propose some ideas to adapt the training to the "digital natives" way of learning.

Palabras clave: pedagogía, traducción e interpretación, TIC, (meta)cognición, socioconstructivismo

Keywords: pedagogy, translation and interpreting, ICT, (meta)cognition, social constructivism 


\section{Introducción}

La pedagogía de la traducción y de la interpretación ha ido evolucionando, desde los años 50 del siglo pasado, a medida que la propia disciplina traductológica se ha ido consolidando y ampliando su foco de análisis. Diferentes planteamientos didácticos han ido matizando el proceso de formación de traductores e intérpretes. La didáctica tradicional de la traducción y los estudios contrastivos de mediados del siglo XX dieron paso al enfoque por objetivos de aprendizaje propuesto por Jean Delisle en los años 80. En los años 90, Amparo Hurtado nos propuso su modelo didáctico basado en el enfoque por tareas y, recientemente, planteó un nuevo acercamiento, el enfoque por competencias, para con ello responder eficazmente a las exigencias del EEES. En cuanto a los actores del proceso pedagógico, hemos asistido asimismo a una clara evolución de la relación docente-discente. Las jerarquías de enseñanza tradicional, eminentemente vertical, dieron paso a modelos de enseñanza-aprendizaje en los que el discente, con la orientación del docente, se convirtió en el protagonista y constructor de su propio conocimiento y aprendizaje. Estos modelos cognitivo-conductistas y socioconstructivistas se combinaron con los modelos metacognitivos y tuvieron como resultado la redefinición de los papeles desempeñados por los actores del hecho pedagógico.

Pese a estas importantes evoluciones, hoy resulta necesario reflexionar en torno al yo-aquí-ahora que se da en todo proyecto formativo, especialmente en un mundo que se está transmutando con tanta rapidez. Centrarse únicamente en el presente resulta tremendamente arriesgado desde un punto de vista pedagógico, por lo puntual y estático de tal perspectiva. El formador de traductores e intérpretes debe tener un ojo puesto en el presente, pero el otro tiene que ir escrutando un horizonte que se va dibujando, aunque tal vez resulte todavía apenas perceptible.

En las siguientes líneas, presentamos una panorámica general de la evolución de la pedagogía de la traducción y de la interpretación para entender de dónde venimos, adónde estamos y hacia dónde nos podríamos estar dirigiendo. 


\section{De la interpretación}

El nacimiento de la interpretación como profesión, (casi) como la conocemos hoy, se concreta al finalizar la I Guerra Mundial. En la conferencia de paz de París (1919), se establece la cooficialidad del inglés y del francés y, por ende, se torna imprescindible la presencia de intérpretes que faciliten la comunicación entre los asistentes a la conferencia. Los intérpretes trabajarán en consecutiva, pues las cabinas de interpretación simultánea, en una versión muy primitiva respecto de lo que hoy conocemos, no verían la luz hasta finales de los años 20 (Baigorri 2000). Dicho esto, la consolidación y generalización de la simultánea no se dará hasta la celebración de los Juicios de Núremberg (1945-1946). Los años 1919 y 1945 marcarán, pues, un antes y un después en la vertebración y consolidación profesional de la interpretación de conferencias. Pero no solo eso. Durante aquella primera mitad del siglo XX, se intuye acertadamente la necesidad de formar a intérpretes competentes que sean capaces de lidiar con los retos históricos a los que estaban llamados a enfrentarse. Y precisamente, para poder responder a dichos desafíos, nacieron las primeras escuelas de intérpretes (y traductores) en Europa. ${ }^{1}$ Si recordamos los principales hitos históricos que fueron jalonando la primera mitad del siglo XX — la firma del Tratado de paz de Versalles y la creación de la Sociedad de Naciones (1919), los Juicios de Núremberg (1945-1946), la fundación de la ONU (1945), la creación de la CECA (1951), de EURATOM y de la CEE (1957) —, y nos fijamos en los años en los que las primeras escuelas de intérpretes abrieron sus puertas u ofertaron programas de interpretación no es difícil establecer un vínculo causal entre la realidad histórica y la subsiguiente respuesta académico-formativa.

Durante varias décadas, las primeras escuelas de intérpretes nutrieron sus clases de docentes que procedían directamente del mercado profesional (institucional), entre otras cosas, porque así lo exigía la AIIC, Asociación Internacional de Intérpretes de Conferencia, creada en 1953. Excelentes intérpretes, sin duda, pero formadores que, dadas las circunstancias y el momento histórico en el que tuvieron que ejercer su función, difícilmente podían contar con una preparación docente previa sobre la que sustentar su pedagogía. Este es el motivo por el que, durante muchas décadas, aquella primera generación de docentes basó su pedagogía en lo que Franz Pöchhacker (2016) denomina

1. Institut für Übersetzen und Dolmetschen (Heidelberg, 1930), ETI (Ginebra, 1941), Universidad estatal pedagógica (Moscú,1942), Universidad de Viena (1943), Universidad de Graz (1946), Universidad de Innsbruck (1946), Universidad de Germersheim (1947), Universidad de Saarbrücken (1948), École de hautes études commerciales (París, 1948), Universidad de Trieste (1954), ESIT e ISIT (París, 1957), ISTI (Bruselas,1958). 
el "apprenticeship approach", consistente en "aprender imitando al maestro". Tras la formación de los primeros intérpretes de conferencia a partir de programas específicos (años 40-60), durante los años 60 y 70 del pasado siglo, las traductólogas francesas, en sus inicios intérpretes de conferencia, Danica Seleskovitch y Marianne Lederer proponen su célebre "Teoría del sentido" que aplicarán a la formación de los futuros intérpretes. Al partir de una reflexión previa, la formación se tornará menos intuitiva por lo que a los objetivos se refiere. Sin embargo, la metodología seguirá siendo muy parecida a la de las décadas anteriores: una metodología marcadamente prescriptivista en la que el protagonista será el docente, que es el "detentador del saber". Así, la relación "maestro-discípulo" seguirá siendo vertical y el discente continuará "imitando al maestro". Los programas pedagógicos se vertebrarán pensando, en las necesidades del mercado (sobre todo, institucional) de la interpretación. Durante muchas décadas, la propuesta docente de Seleskovtich y Lederer gozará de gran popularidad y su célebre Pédagogie raisonnée de l'interprétation, publicada en 1989 y corregida y ampliada en el año 2002, será una de las más influyentes en la historia de la formación de intérpretes de conferencias.

Sin embargo, a partir de los años 80 , la pedagogía sentidista empezará a recibir duras críticas, sobre todo por su limitado poder explicativo a la hora de abordar el proceso y las destrezas necesarias para llevar a cabo una interpretación y por su acentuado prescriptivismo. Será la época en que investigadores cognitivistas como Daniel Gile, Barbara Moser-Mercer o Sylvie Lambert tratarán de dotar de mayor rigor científico a las investigaciones llevadas a cabo en el ámbito de la interpretación, en general, y de su pedagogía, en particular. A tal efecto, partirán del enfoque empírico para proponer modelos explicativos del fenómeno de la interpretación de conferencias. Modelos, como el de los esfuerzos de Gile (1995), que aplicarán a la formación de intérpretes y con los que tratarán de descomponer el proceso (cognitivo) subyacente a la interpretación para con ello trabajar aspectos didácticos fundamentales como son las destrezas interpretativas, las estrategias y las tácticas propias de la interpretación, la gestión de la capacidad de procesamiento o el desarrollo de la pericia en interpretación.

En los años 90, se produce el denominado social turn en el ámbito de la interpretación, lo cual conllevará la reafirmación del interés por la interpretación en los servicios públicos por parte de la comunidad científica. Esto tendrá su reflejo sobre la pedagogía misma de la interpretación en tanto en cuanto se tomará conciencia de un hecho que, de manera tal vez más tácita que explícita, el intérprete profesional ya asumía: la interpretación es una actividad "socialmente situada". Así, en la última década del siglo XX, el estudio y conocimiento 
de los entornos de actuación e interacción del intérprete serán tan importantes como los aspectos cognitivos que subyacen a la actividad interpretativa. Una vez implantadas las formaciones y habiéndose incorporado al mercado laboral las primeras hornadas de intérpretes con una formación específica (primera mitad del siglo XX), es el momento de investigar y de reflexionar acerca de lo que se ha hecho en el ámbito, entre otros, de la formación de intérpretes, sobre lo que se está haciendo y sobre aquello que se debería hacer en el futuro para mejorar dichas formaciones (desde la segunda mitad del siglo XX hasta la actualidad).

Así, durante los años 50 del siglo pasado, se produce el inicio de la investigación en interpretación (de conferencias). Los "investigadores" de aquella época escriben manuales didácticos, sin pretensión científica alguna, basándose en su propia experiencia como intérpretes en activo. A partir de los años 60 y 70 , entran en escena especialistas de otras disciplinas que se interesan por el fenómeno de la interpretación y lo investigan "de manera más científica" desde su propio ámbito de estudio. Tenemos así lingüistas, pero sobre todo psicólogos y psicolingüistas que propondrán experimentos, hoy cuestionados dada la metodología empleada, su discutible validez y lo discreto de los resultados obtenidos, con vistas a investigar la actividad interpretativa. Superados aquellos primeros compases de la investigación, llegamos a la época de la fundamentación académica. Surgen así lo que podríamos denominar las "primeras escuelas", en el sentido científico del término. Dicho esto, no será hasta los años 70 y 80 cuando se sienten realmente las primeras bases de esa cimentación académica. Es la época de los practisearchers, como los denominará Gile (1994), es decir profesionales de la interpretación que también se dedican a investigar. Destacan entre ellos Danica Seleskovitch, Marianne Lederer o Karla Déjean le Féal. Mención especial merecen las dos primeras, Seleskovitch y Lederer, por haber propuesto la previamente citada Teoría del sentido y haber fundado, algunos años antes, la Escuela de París o Escuela del sentido (ESIT). Sus miembros rechazan las investigaciones experimentales de los años previos y las aportaciones de otras disciplinas por considerarlas irrelevantes para la profesión y para la formación. En ese afán por sentar las bases de la investigación traductológica, crean unos de los primeros programas de doctorado en Traductología. En esta época de cimentación académica (años 60-90), tiene lugar, dentro de la lógica de acontecimientos que se habían ido sucediendo, un congreso que supondrá un hito dentro de la historia de la investigación en interpretación. Se trata del Simposio de Venecia, organizado por David Gerver y H. Wallace Sinaiko en 1977 y que trajo consigo la publicación, un 
año más tarde, de las actas que llevarían por título: Language Interpretation and Communication.

Los años 80 y 90 fueron años de apertura a nuevas posibilidades en la investigación de la interpretación. Aparecieron académicos que, lejos de abrazar posturas prescriptivistas y autárquicas, reorientaron la investigación y la abrieron a la interdisciplinariedad. Esta nueva manera de proceder trajo consigo investigaciones más ricas y plurales. En estas mismas décadas se produce una diversificación en los ámbitos y modalidades o géneros abordados por los traductólogos que analizan la interpretación. Así, se empieza a investigar, en un primer momento en Estados Unidos y Canadá, el ámbito de la interpretación en los servicios públicos, siendo la primera de ellas la interpretación judicial, y de la interpretación en lengua de signos. Todo ello exige la exploración de nuevas metodologías, se plantean nuevos paradigmas de investigación y se amplía y se diversifica el foco de análisis tradicional. En 1986, se organiza un simposio sobre la formación de intérpretes que supondría un hecho sin precedentes en la historia de la investigación en pedagogía de la interpretación. La publicación que surgió a raíz de aquel congreso fue la que editaron Laura Gran y John Dodds en el año 1989: The theoretical and practical aspects of teaching conference intepreting. En 1989, se inaugura asimismo el CETRA, Centro especializado en la investigación en Traducción e Interpretación, radicado en Bélgica. Diremos, por último, que en el año 1988 nació en el SSMIT de Trieste (Italia) la primera revista científica especializada dedicada en exclusiva a la interpretación: The Interpreter's Newsletter, la cual sigue vigente.

Llegamos a la última década del siglo pasado. Esta es la época de la consolidación y de la integración de la investigación en interpretación. Efectivamente, asistimos al afianzamiento de los estudios de interpretación (Interpreting Studies) como interdisciplina y a su integración como parte de la Traductología (Translation Studies) con, eso sí, una idiosincrasia muy marcada. El número de publicaciones científicas dedicadas a la interpretación, así como la cooperación entre investigadores aumenta exponencialmente. Se ponen en marcha plataformas de intercambio de información especializada en interpretación como IRTIN/CIRIN, creada por Daniel Gile. En 1996 nace la revista acaso más prestigiosa dedicada monográficamente a la interpretación: Interpreting: international journal of research and practice in interpreting. Además de todo ello, como consecuencia del ya mencionado social turn, se afianza definitivamente el interés por la interpretación en los servicios públicos por parte de la comunidad científica.

Siglo XXI. Ya finales del siglo XX, se reivindicó algo que sabíamos desde hacía tiempo: la interpretación en los servicios públicos era no solo una 
necesidad social, sino una exigencia para respetar ciertos derechos fundamentales del ser humano. Esto no quiere decir que la interpretación de conferencias haya perdido importancia. Simplemente, el espectro profesional se ha ampliado y todavía lo hará más. Ello está teniendo ya un claro reflejo en el terreno de la investigación en la medida en que, en los últimos años, el número de investigaciones dedicadas a la ISP ha superado a las que se han realizado en interpretación de conferencias (Gile 2017). Por otra parte, la vertiginosa evolución y concepción de tecnologías inimaginables hace tan solo un par de décadas repercute directamente sobre la configuración actual de la profesión, de la formación y de la investigación. En este sentido, la idea de la interpretación a distancia no es algo nuevo, pero sí lo son las tecnologías que hoy nos permiten llevarla a cabo. ${ }^{2}$ De hecho, se están utilizando cotidianamente en el terreno profesional tanto en interpretación de conferencias como en ISP. Por ejemplo, en el ámbito sanitario, la interpretación telefónica es cada vez más habitual gracias la aparición de nuevas aplicaciones dedicadas. Las nuevas tecnologías también están teniendo un papel determinante a la hora de preparar, temática y terminológicamente, los encargos de interpretación simultánea. Programas como Interplex, InterpretBank, LookUp, TERMINUS, InterpretersHelp o GlossaryAssistant ayudan a gestionar de manera más eficiente la documentación fuera de la cabina y a hacer consultas rápidas en cabina. Los avances tecnológicos están brindando la posibilidad de explorar y desarrollar nuevas formas de trabajo como, por ejemplo, la consecutiva-simultánea, el respeaking, la interpretación discurso a texto o la interpretación automática (Pöchhacker 2016: 188, 189).

Teniendo en cuenta lo anterior, nos podríamos plantear si las formaciones de intérpretes están o no adaptándose a los cambios sociales y tecnológicos que se están produciendo. Las universidades y sus formadores deben ser conscientes de lo que la sociedad está demandando, de lo que se necesita y adaptarse lo antes posible a ello para dar una respuesta satisfactoria. Es una exigencia profesional, social y humana. Por otra parte, conviene no mostrarse renuentes ante las TIC. Las (nuevas) tecnologías, aun no siendo perfectas, pueden constituir, si se utilizan correctamente, la llave maestra que abra nuevas puertas pedagógicas. En este mismo sentido, cabe recordar que, ya a mediados de los años 90, los formadores de intérpretes se dieron cuenta del interés de utilizar la tecnología, principalmente informática, para mejorar la efectividad de la

2. Cf. la terminología propuesta por Andrew Constable: https://www.isit-paris.fr/wp-content/ uploads/2015/09/ISIT-DGINTER_Synthese_juillet2015.pdf Consultado en noviembre de 2018. 
docencia, así como del aprendizaje autónomo de los intérpretes en ciernes (Sandrelli 2016). Los grandes progresos tecnológicos de principios del siglo XXI, en general, e informáticos, en particular, están facilitando la creación de nuevas herramientas para la formación de intérpretes, las cuales se pueden dividir en dos grandes grupos: 1) las basadas en recursos web; repositorios en línea, principalmente y 2) entornos virtuales para la enseñanza-aprendizaje de la interpretación (también conocidos como Course Management Systems, CMS, o Virtual learning environments, VLE).

$\mathrm{Si}$, a la luz de todo lo hasta aquí expuesto, asumimos que la interpretación es una actividad psicocognitiva y socialmente situada, parece que los métodos de enseñanza tradicionales ya no resultan pedagógicamente (tan) operativos. En este sentido, desde hace algún tiempo se está tratando de aplicar, con las pertinentes adaptaciones, el aprendizaje situado a la formación de intérpretes. En los entornos de aprendizaje situado, el estudiante se enfrentará a situaciones de aprendizaje auténticas en las que deberá, de manera activa y siendo protagonista de la construcción de su propio conocimiento, tal y como defiende el socioconstructivismo, poner en práctica estrategias que le conduzcan a la resolución de problemas concretos. Pero, además, esa resolución no se hará de manera individual o aislada, sino que incluirá al entorno social (esto es, actores de la situación, espacios y tiempos) en el que el estudiante deberá intervenir. Y todo ello con un propósito o una finalidad definida de antemano en un pliego de condiciones. En definitiva, situar el aprendizaje significará ubicar el pensamiento y la acción en un espacio y en un tiempo específicos (esto es fundamental para nosotros cuando sabemos que trabajar en un laboratorio de interpretación puede resultar, a priori, de lo más artificial). Situar el aprendizaje significará incluir a otros estudiantes, incluir el entorno y actividades que resulten «significativas», pues es bien sabido que aprender resulta más factible y efectivo si somos capaces de generar sentido a partir de actividades reales de la vida cotidiana. Situar significa colocar el proceso de pensamiento-acción que los expertos llevan a cabo para realizar tareas que impliquen un saber, un saber hacer y un saber ser en un entorno o situación particular. Por lo tanto, aplicar el aprendizaje situado supone no olvidar ninguno de los elementos básicos, es decir, el contenido, el contexto, la comunidad y la participación. En cuanto al contenido, el aprendizaje situado subraya la necesidad de aplicar acciones derivadas de los procesos cognitivos superiores en situaciones auténticas, pero escogidas con sumo cuidado en función del perfil de los discentes en cuestión. Por lo que al aprendizaje en contexto se refiere, esta teoría defiende la pertinencia de construir un entorno formativo que sea sensible a las tareas que los estudiantes deben ejecutar para conseguir los objetivos establecidos. Al hablar 
de contexto, cabe pensar también en la interacción del estudiante con los valores, las normas, la cultura de una comunidad, en sentido amplio. A través de la comunidad, los estudiantes interpretan, crean y plasman el sentido. Por último, la participación supone un intercambio de ideas a la hora de resolver un problema y un compromiso activo por parte del estudiante respecto de sus compañeros.

La interpretación, del mismo modo que la traducción, no se lleva a cabo a partir de recetas fijas. Su pedagogía tampoco. La pedagogía de la interpretación ya no puede ser ni prescriptiva, ni estática ni jerárquicamente vertical. La alternativa, esencialmente descriptiva, dinámica y jerárquicamente horizontal, radica en una pedagogía descriptivo-constructivista que constituya la vía más directa hacia la metacognición del discente. Se trata, en definitiva, de que el estudiante vaya descubriendo y construyendo su propio conocimiento y extrayendo sus propias conclusiones en ese proceso y que todo ello le ayude a convertirse en un profesional y, sobre todo, en un ser humano íntegro, siempre dispuesto a conocerse a sí mismo en ambas facetas y a mejorar día a día.

\section{De la traducción}

Como comentábamos en las líneas previas, la interpretación y la traducción se cuentan entre las profesiones que más se han transformado en el último medio siglo gracias a los avances de las tecnologías y, por supuesto, a la masificación de las tecnologías de la información y de la comunicación (TIC). Para muchos intérpretes y traductores, el conocimiento de las herramientas tecnológicas utilizadas en sus respectivos campos de actividad se ha convertido en un tipo de llave para poder entrar en las comunidades de práctica profesional. Varios de los artículos incluidos en este número aportan nuevas luces a esta discusión.

Los sistemas de educación se van adaptando a las novedades tecnológicas para con ello garantizar la pertinencia de su oferta educativa. Los avances logrados en las tecnologías asociadas a la traducción automática (TA), a las herramientas de traducción asistida por ordenador (TAO) y las aplicaciones de inteligencia artificial (IA) han transformado la interpretación y la traducción, pero aún están lejos de enviarlas a la lista de profesiones en peligro de extinción. Un caso palpable de esta transformación ha sido la consolidación del fenómeno de la postedición. Las aplicaciones de traducción automática han sido integradas a los procesos de traducción con el fin de responder al aumento en la demanda de traducciones como resultado de los procesos de mundialización (Gambier 2014). Algunos grandes actores del mercado de la traducción en el mundo como la Dirección General de Traducción de la Comisión Europea y La Oficina de traducciones del gobierno canadiense han 
visto en los programas de traducción automática una solución a los problemas que plantea la necesidad de traducir una gran cantidad de documentos en poco tiempo. Los grandes proveedores de servicios de traducción en el mundo han adoptado estas tecnologías provocando cambios sustanciales en el ejercicio profesional de la traducción. El trabajo de un buen número de traductores se transforma de esta manera en el de revisores o poseditores de los textos que producen los programas de traducción automática o que son pretraducidos con memorias de traducción. Esa parte del mercado de la traducción constituye una muestra de la industria en la que deberán trabajar los futuros traductores. Los responsables de la educación de traductores ${ }^{3}$ deben estar al tanto de esta realidad para adaptarse progresivamente a los cambios.

La educación en general vive un fenómeno similar al de las transformaciones que los avances tecnológicos han provocado en el mundo de la traducción y de la interpretación, pero, en este caso, tiene que ver con la transformación del papel de los profesores. Las nuevas generaciones cuentan con grandes facilidades para acceder de manera directa e instantánea a la información y esta realidad influye en la manera de aprender.

La generación de los llamados 'nativos digitales' (Prensky 2001), las personas que nacieron en un mundo en el que ya existían las TIC y sobre todo internet, ha desarrollado su propia manera de aprender. La forma de aprender de los nativos digitales representa un reto para los educadores ya que es bien sabido que los estudiantes deben estar completamente implicados en su proceso de aprendizaje para que la adquisición de conocimientos tenga un efecto duradero. Estudios neurológicos aplicados al aprendizaje lo demuestran. El ser humano solo puede enfocarse en una cosa a la vez y nuestra capacidad para dividir nuestra atención depende de nuestra capacidad de dirigir la atención de una actividad a otra de manera rápida y eficaz mientras hacemos conexiones entre los puntos claves de cualquier actividad que estemos realizando (Bresciani 2016: 10).

El papel de los profesores en el proceso de aprendizaje, como en épocas anteriores, se adaptará con toda seguridad a las características de los nativos digitales. En este mismo sentido, el profesor dejará de ser un intermediario entre los estudiantes y los conocimientos y las habilidades que deben ser adquiridos. Las tecnologías permitirán al alumno interactuar directamente con los conocimientos que serán esenciales para su profesión y los profesores, como siempre lo han hecho, adaptarán su manera de enseñar teniendo en cuenta la

3. Entendemos los conceptos de educación y formación en el sentido propuesto por Widdowson (1984) y Bernardini (2004: 19-20). 
manera en que sus estudiantes conciben y enfrentan el aprendizaje. La educación de traductores no escapará a esta realidad.

Los modelos pedagógicos en los que el aprendizaje debe suceder en un aula en un momento determinado del día y en los que se exige a los estudiantes desconectarse de su mundo virtual el tiempo que dure una lección deberán adaptarse a los cambios sociales que vemos desarrollarse en otros ámbitos como el cine y la televisión en los que los usuarios deciden qué programa van a ver, el momento para verlo y el medio para verlo.

Desde finales del siglo pasado, la educación universitaria en general viene experimentando el paso de un modelo de formación centrado en los profesores y en la enseñanza a un modelo de formación centrado en el aprendizaje y en los estudiantes (Barr \& Tagg 1995). La educación de traductores también ha experimentado este cambio. Como lo confirma Yves Gambier (2012: 163) "[...] we can notice a shift in many places from a teacher-oriented approach to a learner-centered approach, or rather a mixture of approaches." Como veremos a continuación, algunas de las transformaciones más importantes que ha vivido la educación de traductores en los últimos 20 años tienen como denominador común esta idea de centrarse en el aprendizaje y en los estudiantes.

El objetivo de hacer del estudiante el centro de los esfuerzos educativos es un problema complejo que se puede estudiar desde muchas perspectivas. Una de ellas sería enfocarse en el papel activo que cada estudiante debe desempeñar en las actividades de aprendizaje. El aprendizaje activo consiste en implicar cognitivamente a los estudiantes en actividades que involucran algún tipo de "manipulación" cognitiva de los contenidos de los cursos. Cuando se organizan las actividades de aprendizaje, en un enfoque verdaderamente centrado en los estudiantes, se busca implicarlos en procesos cognitivos superiores como la síntesis, el análisis, la evaluación, o la crítica.

Amparo Hurtado Albir fue una de las primeras traductólogas en proponer una estructura didáctica y pedagógica que brindara un marco apropiado para la aplicación de este principio del aprendizaje activo. A finales de los años 90, Hurtado Albir adaptó la fórmula del aprendizaje por tareas a la formación de traductores. Un aspecto clave de la contribución que Hurtado Albir ha hecho a la educación de traductores es haber enriquecido el discurso de la didáctica y de la pedagogía de la traducción con ideas y conceptos propios de las ciencias de la educación en general y de la enseñanza de lenguas extrajeras en particular.

Otros autores emprendieron trayectos similares. Con la publicación de su libro A Social Constructivist Approach to Translator Education (2000), Donald Kiraly dio otro paso importante en la educación de traductores cuando propuso un enfoque de aprendizaje de la traducción fundado en los principios de una 
de las grandes teorías del aprendizaje, el constructivismo social. La propuesta teórica de Kiraly se apoya en el enfoque de aprendizaje por proyectos como medio para llevar esta teoría a la práctica. El gran cambio que introdujo Kiraly en la formación de traductores fue la necesidad de estructurar las propuestas de enseñanza de acuerdo con los preceptos de una teoría del aprendizaje y de proponer una fórmula pedagógica para la aplicación práctica de la teoría.

Antes de Kiraly, el único autor que había apoyado su propuesta didáctica en una teoría del aprendizaje había sido Jean Delisle cuando, en 1980, propuso un método de enseñanza de la traducción apoyado en el enfoque de aprendizaje por objetivos que consiste en la segmentación de los contenidos en pequeñas unidades para que los estudiantes los adquieran progresivamente. Cada pequeña unidad de contenido es considerada como un objetivo de aprendizaje. Delisle también propone una serie de ejercicios para superar lo que él (1981: 10) llamó las "traducciones colectivas" y que Kiraly llamó el método del "who takes the next sentence?" ["¿Quién lee la siguiente frase?"]. Las propuestas didácticas y pedagógicas de Hurtado Albir, de Kiraly y de Delisle comparten pues, entre otras motivaciones, el deseo de superar el método del "¿Quién lee la siguiente frase?"

La idea de superar el método del "¿Quién lee la siguiente frase?" se ha convertido en un lugar común, en un tópico, entre los traductólogos interesados por la didáctica y la pedagogía de la traducción. Se trata de un método de enseñanza de la traducción que aún perdura en muchos contextos. De allí el título de la convocatoria para este número de MonTI y de este artículo introductorio "Porque algo tiene que cambiar". En nuestros días, los responsables de la educación de traductores deben estar al tanto del estado de la cuestión en la enseñanza de la traducción para que su quehacer educativo se apoye en conocimientos compartidos y no exclusivamente en instintos o en experiencias personales.

Los esfuerzos en la formación de formadores de traductores (Kelly 2005) comienzan a tener un impacto sobre la manera de enseñar la traducción. Como en el caso de la traductología en general, el conocimiento sobre la educación de traductores ha progresado considerablemente en los últimos cuarenta años. Estos avances han sido posibles gracias a la consolidación de una comunidad de investigadores que han elegido la educación de intérpretes y traductores como área de investigación. Hoy es posible hablar de esfuerzos de investigación duraderos con los que se han podido lograr niveles de conocimiento avanzados en algunos temas particulares. Un buen ejemplo es el de la competencia traductora. No viene al caso repasar todos los modelos. Para el propósito de nuestra discusión, nos limitaremos a referir a algunos de los trabajos más 
importantes que nos permiten comprobar que los esfuerzos de investigación sobre la competencia traductora han sido continuos durante las cuatro últimas décadas (Wilss 1982; Roberts 1984; Krings 1986; Lörscher 1991; Toury 1995; Pym 2003). Los estudios a los que referimos, entre paréntesis, se caracterizan por ser el producto de un conocimiento experto. Es decir, los autores que los proponen se sirven de su conocimiento en el tema para proponer su idea de la competencia traductora. El conocimiento experto se opone a un conocimiento que podemos llamar, compartido, porque es el que se produce gracias a un programa de investigación y, de alguna manera, es validado por una comunidad científica que comparte los mismos intereses.

El conocimiento experto se caracteriza por ser puntual y por no inscribirse dentro de un programa de investigación sistemático. Así, por ejemplo, el enfoque minimalista de la competencia traductora que propuso Anthony Pym en 2003 es un tipo de conocimiento experto de gran valor en el seno de la comunidad científica pero que no se compara con el conocimiento compartido que ha generado una iniciativa de investigación sistemática como la que han realizado los miembros del grupo PACTE (Proceso de adquisición de la competencia traductora y evaluación) de la Universidad Autónoma de Barcelona desde 1997 hasta nuestros días. Este trabajo de más de 20 años de esfuerzos investigativos permite a toda la comunidad traductológica contar con bases epistemológicas sólidas al momento de discutir sobre el tema de la competencia traductora. Al mismo tiempo, el trabajo de PACTE en torno a la competencia traductora fomenta un diálogo epistemológico en el que otros investigadores o expertos toman posición sobre el tema para dar origen a lo que Imre Lakatos (1978) llamó un programa de investigación científica. En él, tanto las posiciones críticas y paralelas son necesarias para el avance de las ciencias.

Donald Kiraly (2000: 13), por ejemplo, entiende el concepto de competencia traductora como la adquisición de habilidades específicas que permiten al traductor producir un texto en una lengua partiendo de un texto existente en otra lengua. Es claro que Kiraly está utilizando el mismo término que han utilizado otros autores antes y después de él. Pero también es evidente que no se trata del mismo concepto. Esto se puede ver fácilmente si comparamos la definición que ofrecía Amparo Hurtado Albir (2008: 27) de su idea de competencia traductora:

[...] El sistema subyacente de conocimientos declarativos y esencialmente operacionales, necesarios para traducir, con algunas particularidades: (1) es un conocimiento experto que no poseen todas las personas bilingües; (2) es un conocimiento esencialmente operativo y no declarativo; (3) está compuesta por varias subcompetencias interconectadas, (4) la competencia estratégica, 
como es el caso para todo conocimiento operacional, desempeña un papel determinante [Nuestra traducción].

Este tipo de diferencias conceptuales demuestran la complejidad de un concepto clave en la educación de traductores y enriquecen el discurso sobre la traducción y sobre la educación de traductores. Hemos retomado el ejemplo de los conceptos de competencia traductora y de competencia del traductor para resaltar el impacto que los conocimientos compartidos pueden tener en el desarrollo de un área de estudio como la educación de traductores. Hurtado y Kiraly se interesan en un fenómeno similar, pero lo conciben de manera diferente y lo más importante es que lo estudian desde dos perspectivas epistemológicas diferentes. Los trabajos del grupo PACTE y de Hurtado parten de una perspectiva analítica que se interesa por la descomposición del sistema de la competencia traductora en sus elementos constituyentes y que parte de lo complejo para llegar a lo simple. Por su parte, Kiraly utiliza una perspectiva de totalidad. Desde esta perspectiva, la suma de los elementos separados no siempre garantiza la funcionalidad del sistema. La perspectiva totalizante, a su vez, nos privaría de conocer los elementos del sistema ya que solo permite una visión exterior del conjunto y de sus funciones. La teoría de la complejidad defiende la relación dialéctica entre perspectivas epistemológicas opuestas y propone verlas como antagónicas, contradictorias y, al mismo tiempo, complementarias (Morin, 2005: 72). Lo ideal sería que otros temas relativos a la educación de intérpretes y traductores como la motivación para aprender, la evaluación, la retroacción, la investigación en el aula, el papel de las TIC en la educación de traductores, entre muchos otros, fueran objeto de discusión constante y de esfuerzos de investigación sostenidos como han hecho Amparo Hurtado Albir y el grupo PACTE para el caso de la competencia traductora. Además, una parte de los esfuerzos investigativos deberán concentrarse en el estudiante como principal responsable de su propia educación. Entre las ponencias presentadas en el $4^{\circ}$ congreso DidTRAD, realizado en Barcelona en junio de 2018, algunas tenían que ver con conceptos como la autoeficacia, las estrategias de aprendizaje de la traducción, la motivación o la práctica reflexiva. A estos conceptos tendríamos que agregar el concepto de metacognición. La metacognición entendida como el conocimiento que poseemos de nuestras capacidades cognitivas y como el control que podemos ejercer sobre los procesos cognitivos. Por ejemplo, conocer los límites de nuestra capacidad para retener algún tipo de información (cifras, caras, lugares, palabras, nombres de personas) es un buen ejemplo de conocimiento metacognitivo. Cuando estamos leyendo y nos damos cuenta de que no estamos concentrados estamos controlando el proceso de comprensión de lectura. Estos conceptos constituyen 
un conjunto de conocimientos que los educadores debemos compartir con los estudiantes con el fin de que ellos puedan ejercer un mejor control sobre su aprendizaje.

Aprender a aprender y aprender para la vida se han convertido en dos lemas claves de la educación, pero en muchos casos los educadores no cuentan con las herramientas conceptuales necesarias para la concretización de esos dos lemas en su práctica cotidiana. Encontrar un espacio dentro de las actividades de aprendizaje para que los estudiantes se familiaricen con conceptos como la metacognición, la motivación, la autoeficacia y las estrategias de aprendizaje se convierte en un reto interesante para la investigación a corto y mediano plazo en la educación de traductores. La educación de traductores verdaderamente centrada en los estudiantes empieza por dirigirlos hacia estos conceptos para que ellos ejerzan un mejor control sobre su aprendizaje. Además de aprender a traducir y de aprender sobre la traducción, el futuro traductor deberá aprender sobre el aprendizaje para aprender a aprender.

\section{Consideraciones finales}

La evolución de la pedagogía en el ámbito de la traducción y de la interpretación ha sido, y sigue siendo, particularmente dinámica. Dos parecen haber sido los motores de tal dinamismo desde los años 40 del pasado siglo hasta nuestros días: por un lado, los acontecimientos diplomáticos, sociales, culturales y económicos que fueron jalonando la historia del siglo XX y XXI y por otro, el desarrollo y democratización de ciertas tecnologías de la información y de la comunicación. Tal y como hemos visto en las líneas previas, las exigencias profesionales trajeron consigo la creación y generalización de centros especializados en la formación de traductores e intérpretes. Las formaciones en esos centros empezaron siendo, como no podía ser de otro modo dadas las circunstancias, muy intuitivas para, poco a poco, irse sofisticando gracias, también, al planteamiento y desarrollo de investigaciones pedagógicas de profundo calado. Así, de la pedagogía centrada en el docente, pedagogía jerárquicamente vertical, pasiva y estática, se pasó a la pedagogía centrada en el verdadero protagonista de cualquier proyecto pedagógico: el estudiante. Una pedagogía horizontal, activa y dinámica en la que el docente ya no "enseña", sino que "modera" y se convierte en "facilitador". Facilita a los estudiantes herramientas y situaciones necesarias para que éste pueda construir su propio conocimiento y esté en condiciones de emprender con garantías el viaje hacia su autoconocimiento como profesional y como persona.

Investigaciones, aquellas, que fueron absolutamente necesarias e inspiradoras, como, esperemos que lo sean, las que el lector encontrará en el presente 
número de MonTI. Contribuciones que vienen a arrojar nueva luz sobre el ayer, el hoy y, sobre todo, el mañana de la pedagogía de la traducción y de la interpretación.

\section{Referencias bibliográficas}

BAIGORRI, Jesús. (2000) La interpretación de conferencias: el nacimiento de una profesión, de París a Núremberg. Granada: Comares.

BARR, Robert \& John TAGG. (1995) "From teaching to learning: A new paradigm for undergraduate education." Change 27:6, pp. 12-25.

BERNARDINI, Silvia. (2004) "The theory behind the practice: Translator training or translator education?" En: Malmkjaer, Kirsten (ed.) 2004. Translation in undergraduate degree programmes. Ámsterdam \& Filadelfia: John Benjamins, pp. 17-29.

BRESCIANI, Marilee. (2016) "Introduction: Rethinking how we design, deliver, and evaluate higher education." En: Bresciani, Marilee (ed.) 2016. The neuroscience of learning and development: enhancing creativity, compassion, critical thinking, and peace in higher education. Sterling (VA): Stylus Publishing/ACPA, pp. 1-26.

DeLISLE, Jean. (1980) L'analyse du discours comme méthode de traduction. Ottawa: Presses Universitaires d'Ottawa.

GAMBIER, Yves. (2012) "Teaching Translation/Training Translators." En: Gambier, Yves \& Luc van Doorslaer (eds.) 2012. Handbook of Translation Studies 3. Ámsterdam \& Filadelfia: John Benjamins, pp. 163-171.

GAMBIER, Yves. (2014) "Changing landscape in translation." International Journal of Society, Culture \& Language 2:2, pp. 1-12.

GILE, Daniel. (1994) "Opening up in Interpretation Studies." En: Snell-Hornby, Mary, Franz Pöchhacker \& Klaus Kaindl (eds.) 1994. Translation Studies: An interdiscipline. Ámsterdam \& Filadelfia: John Benjamins, pp. 149-158.

GiLE, Daniel. (1995) Regards sur la Recherche en Interprétation Simultanée. Lille: Presses Universitaires de Lille.

GILE, Daniel. (2017) "Traditions and innovation in Interpreting Studies: a personal analysis for 2016." Domínios de Lingu@gem 11:5, pp. 1424-1439.

HURTADO ALBIR, Amparo. (1996) "La enseñanza de la traducción directa 'general'. Objetivos de aprendizaje y metodología." En: Hurtado Albir, Amparo (coord.) 1996. La enseñanza de la traducción. Castelló de La Plana: Servei de Publicacions de la Universitat Jaume I, pp. 31-56.

HURTADO ALBIR, amparo. (Dir.) (1999) enseñar a traducir. Metodología en la formación de traductores e intérpretes. Madrid: edelsa.

Hurtado AlBiR, Amparo. (2008) "Compétence en traduction et formation par compétences." TTR: Traduction, Terminologie, Rédaction 21:1, pp. 17-64.

KelLy, Dorothy. (2005) A Handbook for Translator Trainers. Manchester: St. Jerome. 
Kiraly, Donald. (2000) A Social Constructivist Approach to Translator Education. Manchester: St. Jerome.

KRINGS, Hans P. (1986) "Translation problems and translation strategies of advanced German learners of French (L2)." En: House, Juliane \& Shoshana Blum-Kulka (dirs.) 1986. Interlingual and intercultural communication. Tubinga: Gunter Narr, pp. 263-275.

LAKATOS, Imre. (1978) The methodology of scientific research programmes. Cambridge: Cambridge University Press.

LÖRSCHER, Wolfgang. (1991) Translation performance, translation process, and translation strategies: A psycholinguistic investigation. Tubinga: Gunter Narr Verlag. MORIN, Edgar. (2005) Introduction à la pensée complexe. París: Éditions du Seuil.

PÖCHHACKER, Franz. (2016) Introducing Interpreting Studies. Londres \& Nueva York: Routledge

PRENSKY, Marc. (2001) "Digital Natives, Digitale Inmigrants. Part 1." On the Horizon 9:5, pp. 1-6.

PYM, Anthony. (2003) "Redefining translation competence in an electronic age." Meta 48:4, pp. 481-497.

ROBERTS, Roda. (1984) "Compétence du nouveau diplômé en traduction, traduction et qualité de langue." Actes du Colloque, Société des Traducteurs du Québecl Conseil de la langue française. Québec: Éditeur officiel du Québec, pp. 172-184. SANDRELLI, Annalisa. (2016) "Becoming an interpreter: The role of computer technology." MonTI, Special Issue 2, pp. 111-138.

TOURY, Gideon. (1995) Descriptive translation studies - and beyond. Ámsterdam \& Filadelfia: John Benjamins.

Widdowson, Henry. (1984) Explorations in applied linguistics 2. Oxford: Oxford University Press.

WILSS, Wolfram. (1996) Knowledge and skills in translator behavior. Ámsterdam \& Filadelfia: John Benjamins. 


\section{NOTA BIOGRÁFICA / BIONOTE}

Miguel Tolosa IguAladA es doctor en Traducción e Interpretación (2009), Máster en Traducción (2004) y licenciado en Traducción e Interpretación por la Universidad de Alicante (2001). Es traductor jurado de francés desde el año 2002. Sus principales líneas de investigación son la traducción y la interpretación como proceso cognitivo y su relación con la resolución de dificultades y con la comisión de errores, la didáctica de la traducción y de la interpretación, la traducción y la interpretación en las organizaciones internacionales. Desde 2005, es profesor en el Departamento de Traducción e Interpretación de la Universidad de Alicante, actividad que combina con la de intérprete y traductor profesional (desde el año 2001). Imparte clases de traducción general y especializada, de interpretación consecutiva y simultánea (Grado en Traducción e Interpretación, UA), de interpretación en los organismos internacionales (Máster en Traducción Institucional, UA) y un seminario de doctorado sobre investigación empírica en el ámbito de la traducción y de la interpretación (Doctorado: Traductología, traducción profesional y audiovisual).

Miguel Tolosa Igualada holds a PhD in Translation Studies (2009), Master's in Translation Studies (2004) and bachelor's degree in Translation and Interpreting (2001), University of Alicante (Spain). He is a sworn translator (French-Spanish/Spanish-French) since 2002. His main research interests are: the translation and the interpreting activities analysed as cognitive processes and the link with difficulty solving and commission of errors; the translation and the interpreting didactics; the translation and interpreting activities within the International Organizations and in the private market. He teaches translation and interpretation at the University of Alicante since 2005. He has been a professional translator and interpreter since 2001. He teaches general and specialized translation, consecutive and simultaneous interpretation (Bachelor's Degree in Translation and Interpreting, UA), Interpreting in International Organizations (Master's Degree in Institutional Translation, UA) and a doctoral course on translation and interpreting empirical research (Doctorado: Traductología, traducción profesional y audiovisual).

ÁlVARO ECHEVERRI es doctor en Traductología (2008) de la Université de Montréal, es Máster en Traducción (2001) de Kent State University (EE. UU.) y es licenciado en Lenguas modernas (1994) de la Universidad de Antioquia (Colombia). Desde 2010 es profesor del Departamento de lingüística y de traducción de la Université de Montréal. Sus líneas de investigación son la pedagogía de la traducción, la traducción de la traductología y la historia de la 
traducción en América latina en la época de la independencia. En lo referente a la pedagogía de la traducción, sus intereses de investigación se sitúan en la formación centrada en el estudiante y en el aprendizaje activo y, particularmente, en la dimensión metacognitiva de la traducción. Su interés por la historia de la traducción se centra en la traducción de textos políticos en la época de la independencia. Imparte clases de documentación para traductores, metodología de la traducción, corrientes teóricas de la traducción y un seminario de formación de formadores en traducción.

Álvaro ECheVerri holds a Ph.D. in Translation Studies (2008) from the Université de Montréal, a M.A. in Translation (2001) from Kent State University (USA) and a B.A. in Modern Languages (1994) from the Universidad de Antioquia (Colombia). His main research interest areas are translation pedagogy, translation of translation studies' seminal texts, and the history of translation in Latin America. Concerning the pedagogy of translation his research in based on student-centred approaches to learning, active learning, in particular the metacognitive dimension of translation. His interests in the history of translation hover around the translation of political texts at the time of Independence. He teaches documentary research for translator, translation methodology, theoretical approaches to translation and a seminar on Translator trainers. 